\title{
COLiDeR: A Cross-Layer Protocol for Two-Path Relaying
}

Raphaël Naves ${ }^{1}$, Gentian Jakllari², Hicham Khalifé1 ${ }^{2}$ Vania Conan $^{1}$, André-Luc Beylot ${ }^{2}$

\begin{abstract}
In this work, we present COLiDeR, the first PHY/MAC cross-layer protocol for practical twopath relaying using off-the-shelf half-duplex radios. It relies on three main contributions. First, based on an off-line performance comparison, COLiDeR selects the best interference management technique for a radio to handle two overlapping signals depending on the measured channel conditions. Then, considering the real decoding capacities of the nodes, COLiDeR introduces a dynamic relaying strategy with the objective of achieving high throughput while reducing decoding failures. This includes a light-weight protocol for the source to evaluate the channel state, a state-machine modeled approach driven by the source for switching between the defined scheduling schemes and an optional power adaptation mechanism for reducing the packet losses. Finally, COLiDeR comes with an adapted scheduling mechanism that aims to integrate two path-relaying in multi-hop wireless networks. Experiments on a 4-USRP testbed show that COLiDeR delivers between 80-95\% of the relaying performance of an ideal full-duplex radio while incurring negligible decoding failures. Just as important, large-scale simulations show that COLiDeR improves network throughput in multihop topologies by over $20 \%$ compared to traditional interference-free transmissions.
\end{abstract}

\section{Introduction}

\subsection{Two-path relaying in a nutshell}

A basic underlying principle of current wireless networks is that a radio cannot transmit and receive at the same time, i.e., it is half-duplex, significantly limiting the performance of in-band relay networks. Consider the simple 4-node network depicted in Fig. 1. The source node, $S$, wants to send a batch of packets to the destination, $D$, which is too far to receive the packets via a direct

\footnotetext{
${ }^{1}$ Thales Communications \& Security - France, Email: name.surname@thalesgroup.com

${ }^{2}$ IRIT-INPT/ENSEEIHT - France, Email:name.surname@enseeiht.fr

${ }^{3}$ An early version of this work appeared as a short paper in the Proc. of the 22nd International ACM Conference on Modeling, Analysis and Simulation of Wireless and Mobile Systems (MSWiM), 2019 [1] 


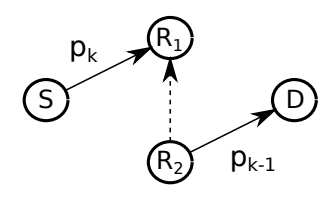

(a) Slot $\mathrm{k}$

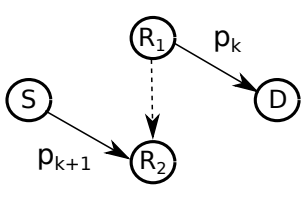

(b) Slot $\mathrm{k}+1$

Figure 1: Two-path relaying. The source, $S$, transmits on every slot, alternating between the two relays, $R_{1}$ and $R_{2}$. When a relay is receiving from the source, the other transmits to the destination, jointly creating the equivalent of a single full-duplex relay.

transmission. Traditionally, $S$ would rely on either $R_{1}$ or $R_{2}$ (both in the range of $S$ and $D$ ) to relay its packets to the destination, node $D$. With half-duplex radios, $S$ and the relay, say $R_{1}$, would have to take turns transmitting, meaning the end-to-end capacity would be at most half the link capacity - a significant penalty, also known as the multiplexing loss. If $R_{1}$ were to have a full-duplex radio, it would be able to transmit to the destination while receiving from the source, eliminating the $1 / 2$ loss factor. The fundamental challenge in this scenario is that $R_{1}$ 's own signal would be hundreds of thousands of times stronger than the signal from $S$. It makes the task of decoding $S^{\prime}$ transmission extremely challenging, explaining the difficulty of realizing a full-duplex radio $[2,3]$.

A far easier task, however, is for $R_{1}$ to decode the transmission from $S$ while another remote station, say $R_{2}$, is transmitting. The powers of two remotely transmitted signals are likely to only differ by several times, paving the way for two-path relaying [4]. Assuming a TDMA (Time-Division Multiple Access) channel access protocol for ease of presentation, the source, $S$, can transmit on every slot, alternating between two relays. As depicted in Fig. 1, in slot $k, S$ transmits a packet to $R_{1}$. In slot $k+1, R_{1}$ forwards the packet to the destination, $D$, while $S$ transmits a second packet, this time to $R_{2}$. If $R_{2}$ can successfully decode the packet from $S$, despite the interference from $R_{1}$, it will forward it to $D$ in the following slot, while $S$ will transmit a third packet to $R_{1}$. Thus, as long as one relay can decode a packet from the source while the other is transmitting a packet to the destination, two-path relaying can eliminate the $1 / 2$ loss factor, achieving the same performance as single-path relaying with a general-purpose full-duplex radio. 


\subsection{Two-path relaying in practice: main challenges}

While two-path relaying has been studied theoretically $[5,6]$, realizing it and making it practical for current multi-hop wireless networks faces three main challenges:

- PHY-Challenge - Interfering packets decoding: Since two-path relaying requires a relay to successfully decode a transmission from the source when the other relay is transmitting, the best interference management technique to handle colliding packets needs to be identified. Many theoretical works $[4,5,6]$ relied on the well-known concept of Successive Interference Cancellation (SIC) for decoding at the relays. It assumes that the inter-relay channel is very strong, allowing the interference to be decoded first and then eliminated from the received signal, leaving only the signal from the source. However, in a practical system, there is no guarantee as to the inter-relay channel, especially if the nodes are mobile.

- REL-Challenge - Intra-diamond relaying: Depending on source-relay and inter-relay channel - we refer to for the rest of the paper as intra-diamond channel - and the decoding performance at relays, two-path relaying is not always feasible in practice since collisions between packets may lead to a high packet loss rate. Therefore, as proposed in [7], a dynamic relaying mechanism alternating between two-path relaying, when error-free decoding at relays is possible, and traditional one-path relaying, otherwise, is necessary. The question, however, of how the stations discover the environment, select and activate the best relaying strategy to use in practice is still open.

- MAC-Challenge - Diamond channel reservation: Most papers are limited to a feasibility study of two-path relaying on the simple 4-node scenario. In larger wireless networks with multiple competing traffic flows, however, two-path relaying requires a specific channel reservation method. The main difference from traditional methods is that 4 nodes (a source, two relays and a destination) have to cooperate to access the channel, instead of two (one source and one destination) as is the case in classical point-to-point communications. 


\subsection{Our contributions}

We present COLiDeR, a novel PHY/MAC CrOss-Layer protocol for practical decode-andforward Diamond ${ }^{4}$ Relaying. COLiDeR builds on two key contributions, addressing the PHYChallenge and the REL-Challenge. To answer the MAC-Challenge, we design a realistic access method on top of COLiDeR.

- PHY: We lead a testbed-driven evaluation and analysis of the best interference management technique for a relay to decode a packet from the source node while the other relay is transmitting a packet to the destination. Among the tested candidates, our experimental study shows that interference-free equalization and Physical-Layer Network Coding [8], another interference management technique, outperforms Successive Interference Cancellation (SIC). Nevertheless, beyond this unexpected result, this comparison of decoding capacity also shows that no solution works best in all channel conditions and that two-path relaying is not always feasible using off-the-shelf hardware. Thus, we introduce the concept of channel-aware decoding areas and use a measurement-driven approach to define their boundaries.

- REL: Building on the decoding areas, we design COLiDeR, a cross-layer protocol capable of identifying the relaying strategy that increases throughput while reducing packet losses due to decoding failures. COLiDeR is a four-pronged source-centered solution: a signaling protocol for the source to ascertain the intra-diamond channel state, a state-machine modeled approach driven by the source for switching between states, a distinct scheduling for every state and an optional power adaptation mechanism for limiting the packet losses at relays. As most wireless communication protocols, COLiDeR introduces different parameters that we left open. However, in order to set up COLiDeR in practice, we lead intensive measurement analysis to discuss the influence of each parameter and select the optimal values. Then, we implement COLiDeR on the USRP radio using a generic QPSK (Quadrature Phase-Shift Keying) modulation with the GNU Radio framework [9], and evaluate its performance on a 4-node testbed using over-the-air transmissions exclusively. The experimental results show that COLiDeR delivers between $80-95 \%$ of the relaying performance of an ideal full-duplex radio while incurring negligible decoding failures.

\footnotetext{
${ }^{4}$ Due to the geometrical shape of the Fig. 1 topology, two-path relaying is also known as a diamond relay network.
} 
- MAC: We introduce a fully-distributed access method running over COLiDeR that aims at reserving the channel for each diamond involved in large wireless networks with multiple nodes and multiples flows. The proposed mechanism is inspired from a well-studied slotted version of the 802.11 DCF protocol, traditionally used for channel reservation of point-to-point communications. We implement the COLiDeR-adapted channel reservation in a discrete-event simulator and evaluate it over a large network topology with different communication patterns. The data shows that two-path relaying can increase the overall throughput of a 50 -node network by $20 \%$.

The remainder of the paper is organized as follows: In Section 2, we carry the experimental evaluation of physical-layer decoding approaches and introduce the channel-aware decoding areas (PHY-Challenge). In Section 3, we design and evaluate COLiDeR (REL-Challenge). In Section 4, we introduce and evaluate the access method that aims to integrate of COLiDeR (MAC-Challenge) in multihop wireless networks. Section 5 gives an overview of the existing works on two-path relaying and section 6 concludes the paper.

\section{Simultaneous reception of multiple packets: A measurement-driven analysis}

In this section, we carry out an experimental study on the feasibility of two-path relaying and the best strategy for relays to handle the simultaneous reception of two signals.

\subsection{Problem formulation}

To simplify the presentation, we consider one part of two-path relaying as depicted in Fig. 1(a). $S$ transmits a packet, $p_{S}$, to $R_{1}$ while $R_{2}$ transmits a packet, $p_{R_{2}}$, to the destination, with $R_{1}$ facing the challenge of decoding $p_{S}$. All the results shown apply to the second part, taking place in the subsequent slot, as depicted in Fig. 1(b).

We consider that packets are sent using an OFDM coding structure with a QPSK symbol mod-

ulation on each subcarrier. Denoting with $x_{S}^{m, k}$ and $x_{R_{2}}^{m, k}$ the symbol transmitted simultaneously on the $m$-th OFDM symbol and the $k$-th subcarrier by nodes $S$ and $R_{2}$, respectively, the symbol $y^{m, k}$ received by $R_{1}$ can be expressed as follows:

$$
y^{m, k}=h_{S R_{1}}^{m, k} x_{S}^{m, k}+h_{R_{2} R_{1}}^{m, k} x_{R_{2}}^{m, k}+n
$$




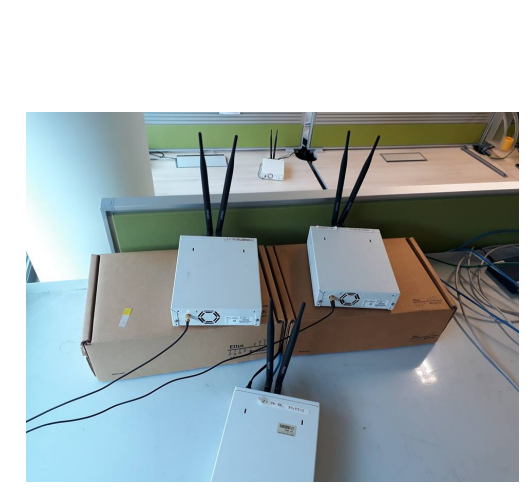

(a)

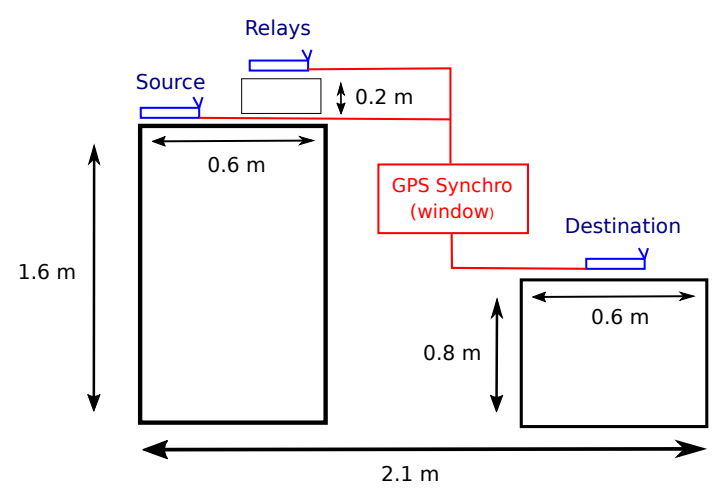

(b)

Figure 2: USRP testbed used for implementing and evaluating diamond-based relaying.

where, $h_{X Y}^{m, k}$ is the channel coefficient of the $m$-th OFDM symbol on the $k$-th subcarrier between $X$ and $Y$ nodes and $n$ is the ambient noise.

Obviously, the symbol $x_{S}^{m, k}$ is the signal of interest for $R_{1}$.

\subsection{The decoding candidates}

We consider three decoding techniques that could potentially be applied at $R_{1}$.

1) Interference-Free (IF) equalization [4][7]: $R_{1}$ decodes directly the packet $p_{S}$ considering the part $h_{R_{2} R_{1}}^{m, k} x_{R_{2}}^{m, k}+n$ of Eq. (1) as Gaussian noise. In particular, $R_{1}$ estimates the symbol $\hat{s}_{S}^{m, k}$ sent by $S$ on the $k$-th subcarrier of the $m$-th OFDM symbol using the minimum distance criteria.

2) Successive Interference Cancellation (SIC) [4][7][6]: This decoding technique is used to decode many packets received simultaneously with different power levels. The principle is to decode the strongest one with an IF equalization and then to cancel its contribution in equation (1), repeating the operation until the signal of interest is decoded [10].

When $S N R_{R_{2}, R_{1}}>S N R_{S, R_{1}}{ }^{5}$, SIC equalization becomes a two-step decoding process. $R_{1}$ estimates the symbol $\hat{s}_{R_{2}}^{m, k}$ sent by $R_{2}$, removes its contribution from the the received signal and finally it estimates $\hat{s}_{S}^{m, k}$ with second IF equalization as follows:

$$
\hat{s}_{S}^{m, k}=\underset{s \in S(Q P S K)}{\operatorname{argmin}}\left|\left(y^{m, k}-\hat{h}_{R_{2} R_{1}}^{m, k} \times \hat{s}_{R_{2}}^{m, k}\right)-\hat{h}_{S R_{1}}^{m, k} \times s\right|^{2}
$$

with $S(Q P S K)$ the 4 symbols composing a QPSK modulation, and $\hat{h}_{R_{2} R_{1}}^{m, k}$ and $\hat{h}_{S R_{1}}^{m, k}$ the channel coefficients estimated at $R_{1}$.

\footnotetext{
${ }^{5} S N R_{X, Y}$ denotes the Signal-To-Noise Ratio of a signal sent by node $X$ measured at node $Y$.
} 
Obviously, when $S N R_{S, R_{1}}>S N R_{R_{2}, R_{1}}$, SIC falls back to a simple IF decoding in which $R_{1}$ aims to retrieve $\hat{s}_{S}^{m, k}$ directly.

3) Physical-Layer Network Coding (PLNC): Never before associated with two-path relaying, we make it the third candidate because it is designed to benefit from two interfering signals. In its simplest form, PLNC [8] consists of decoding a linear combination (typically a XOR combination) of two interfering packets rather than the two individual packets. In the considered scenario for instance, $R_{1}$ aims to decode a linear combination of $p_{S}$ and $p_{R_{2}}$ rather than $p_{S}$ only. However, with a QPSK modulation, decoding the xor-ed packet, $p_{S} \oplus p_{R_{2}}$, is not the optimal solution [11]. Contrary to BPSK, for any modulation of 4-ary cardinality, the best denoising map depends on $\theta^{m, k}=\arg \left(\frac{\hat{h}_{R_{2} R_{1}}^{m, k}}{\hat{h}_{S R_{1}}^{m, k}}\right)$, the phase between the two interfering signals. Let us denote with $\left(\hat{s}_{S}^{m, k}, \hat{s}_{R_{2}}^{m, k}\right)$, the solution to the following minimum distance equation:

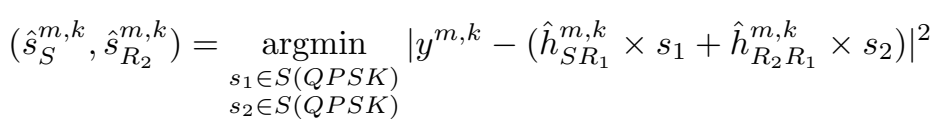

If $\tan \left|\theta^{m, k}\right|<1, R_{1}$ estimates the symbol $\hat{s}_{S}^{m, k} \oplus \hat{s}_{R_{2}}^{m, k}$; otherwise, it estimates $\hat{s}_{S}^{m, k} \oplus \operatorname{Rot}\left(\hat{s}_{R_{2}}^{m, k}\right)$, where $\operatorname{Rot}(x)$ is the anti-rotation function on QPSK symbols given by the following equation:

$$
\operatorname{Rot}(x)=x e^{-j \Pi / 2}
$$

In order to apply the same decoding estimation for symbols belonging to the same packet, we consider a unique $\theta$ value for the whole payload. It is computed by averaging the phase difference obtained in the first OFDM symbol. This decoding technique is known to be optimal in the sense of the minimum distance criteria regardless of the channel conditions [12].

Note that, with PLNC, $R_{1}$ decodes a linear combination of $p_{S}$ and $p_{R_{2}}$ rather than the individual packet $p_{S}$. The destination, using previously received packets (the very first is a single native packet) can perform the PLNC decoding.

\subsection{IF vs. SIC vs. PLNC}

Experimental platform: To compare the performance of the three decoding techniques, we use the testbed depicted in Fig. 2. It consists of 4 Ettus Research Universal Software Radio Peripherals (USRPs) N210 equipped with an SBX daughterboard and GPS antennas. Communications take place over the $1.8 \mathrm{GHz}$ frequency band with a sampling rate of $400 \mathrm{kB} / \mathrm{s}$ and the signal/MAC processing is done in $\mathrm{C}++$ and Python within the GnuRadio framework [9]. A slotted TDMA channel access is built thanks to the GPS synchronization. 
Implementation details: Packets are 1280 bit-long and each OFDM symbol includes 64 subcarriers: 40 subcarriers for data symbols, 12 guard subcarriers and 12 pilot subcarriers. To estimate the channel coefficients $\hat{h}_{R_{2} R_{1}}^{m, k}$ and $\hat{h}_{S R_{1}}^{m, k}$ each interfering payload transmission is preceded by two interference-free preambles sent by $S$ and $R_{2}$ successively [13]. In addition, $R_{1}$ tracks the channel variation thanks to the known symbols on the pilot subcarriers, distributed in orthogonal fashion between $S$ and $R_{2}$. Then, the data subcarriers channel coefficients are obtained thanks to a linear interpolation [14].

Experiment description: To evaluate $R_{1}$ 's capability to decode the signal from the source under diverse intra-diamond channel conditions, we keep the inter-relay link stable and vary the source transmission power. Specifically, we start by modulating the transmission power of $R_{2}$ so as to obtain $S N R_{R_{2}, R_{1}}=30 \mathrm{~dB}$. Leaving $R_{2}$ 's transmission power unchanged for the rest of the experiment, we vary the transmission power of node $S$ and evaluate the Bit Error Rate (BER) at $R_{1}$. When PLNC decoding is applied, the BER is obtained by comparing the decoded packet to $p_{S} \oplus p_{R_{2}}$ or $p_{S} \oplus \operatorname{Rot}\left(p_{R_{2}}\right)$, depending on the $\theta$ value obtained at $R_{1}$.

Results: Figure 3 delivers two main lessons. First and probably most surprisingly, contrary to what is recommended by almost all literature on the subject $[4,6,7]$, SIC is a poor enabler of two-path relaying. Instead, the data shows that on off-the-shelf hardware, using PLNC yields an acceptable BER $\left.\left(<10^{-2}\right)\right)$ for far more intra-diamond channel values.

The second lesson is that depending on the intra-diamond channel state, there are three distinct areas relevant to two-path relaying. When $S N R_{S, R_{1}}>S N R_{R_{2}, R_{1}}$ by more than $5 \mathrm{~dB}$ (right part of the curve), $R_{1}$ can easily decode $p_{s}$ by using IF, as expected. Second, when the difference in SNR between the two signals is between $(5 d B,-15 d B)$ - a wide region - using PLNC enables $R_{1}$ to decode with a reasonable BER. Finally, when the desired signal becomes too weak $(<15 d B)$, the BER starts reaching unacceptable levels.

\subsection{Summary}

The empirical study of this section leads to two main conclusions which will drive the protocol design and evaluation for the rest of the paper. First, we showed that unlike what is commonly believed in literature $[4,6,7]$, a decoding technique based on Physical-Layer Network Coding clearly outperforms Successive Interference Cancellation.

Second, our measurement data showed that the performance of two-path relaying will highly 


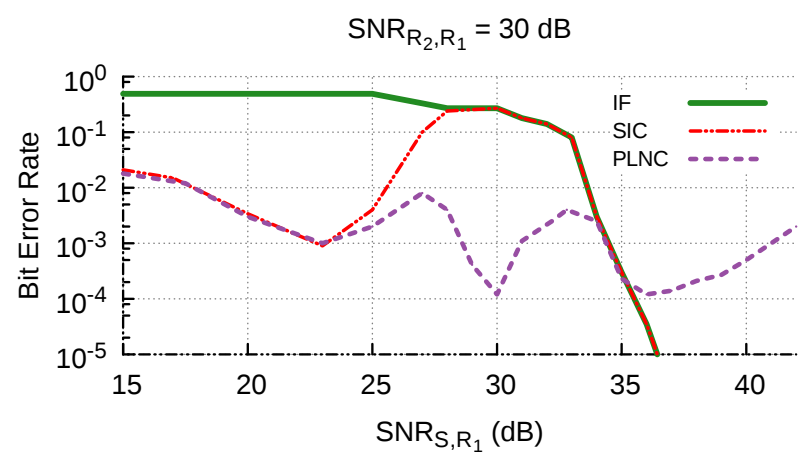

Figure 3: Error Rate at $R_{1}$ with different values of $S N R_{S, R_{1}}$

depend on the intra-diamond channel (IDC). Thus, we identify three IDC-level decoding areas, depicted in Fig. 4, which could lead, as shown in $\S 3$, to different decoding strategies at relays:

- Area $\mathbf{A}$ - the desired signal is stronger than the interfering signal. Using interference-free equalization (IF), the relay, say $R_{1}$ (resp. $R_{2}$ ), can decode the packet from the source while the other relay, $R_{2}$ (resp. $R_{1}$ ), is transmitting a packet to the destination. Therefore, the source and $R_{2}$ (resp. $R_{1}$ ) can transmit simultaneously.

- Area B - the desired signal is similar in strength to the interfering signal. Using PLNC-based decoding, the relay, say $R_{1}$ (resp. $R_{2}$ ), can decode the xor-ed packet containing the packet from the source while the other relay, $R_{2}$ (resp. $R_{1}$ ), is transmitting a packet to the destination. Therefore, the source and $R_{2}$ (resp. $R_{1}$ ) can transmit simultaneously.

- Area $\mathbf{C}$ - the desired signal is significantly weaker than the interfering signal. In this case, there is no known approach for one of the relays, say $R_{1}$ (resp. $R_{2}$ ), to decode the desired signal coming from the source, if the other relay, $R_{2}$ (resp. $R_{1}$ ) transmits to the destination at the same time. These transmissions have to be orthogonalized.

\section{COLiDeR: Design \& evaluation}

In this section, we introduce COLiDeR, a CrOss-Layer Diamond Relaying protocol, whose design is driven by the measurement study presented in $\S 2$. The goal is to achieve two complementary objectives: maximizing the received throughput at the destination while minimizing the number of packet losses due to decoding failures.

COLiDeR faces three key challenges in realizing two-path relaying. First, the definition of the 


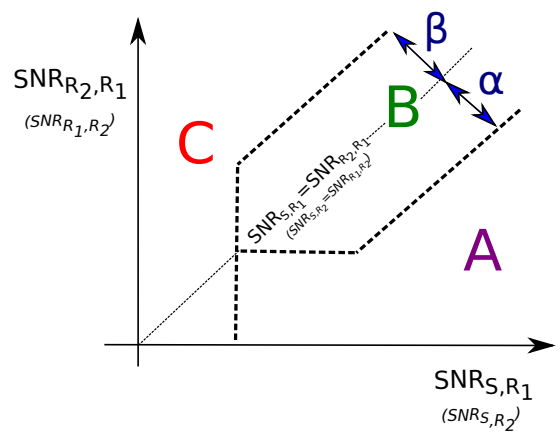

Figure 4: Three decoding strategies at $R_{1}$ depending on the intra-diamond channel (IDC)

decoding areas, A, B, C, in $\S 2.4$ was given in qualitative terms - the exact SNR-based definition of each area is required for the proper functionality of COLiDeR (§ 3.4). Second, assuming the decoding areas are known, specific scheduling policies are necessary, especially for the cases when one relay is able to handle two signals while the other only one (§3.1). Finally, the three nodes will have to agree on which schedule to use in a distributed setting using wireless communications which are inherently lossy $(\S 3.2)$.

In the following, we describe how COLiDeR addresses each challenge, starting by assuming that the decoding areas are known. In $\S 3.4$, we show how COLiDeR relaxes this assumption by using a measurement-driven approach.

\subsection{IDC-aware states and scheduling}

As our measurement study in Sec. 2 demonstrated, the performance of two-path relaying depends on the intra-diamond channel (IDC). To successfully navigate the state of the diamond, COLiDeR identifies 5 states: for three of them scheduling is trivial while for the other two a new scheduling approach is introduced.

\subsubsection{States 0 and 0 '}

None of the relays can decode a packet transmitted from the source, $S$, while the other relay is transmitting - area $C$ in Fig. 4 for the two relays. In this case, COLiDeR relays packets through $R_{1}$ only (state 0 ) or $R_{2}$ only (state 0 '), following the traditional one-path relaying approach. As a result, the source can send at most 1 packet every 2 time slots, for a 0.5 frames/slot maximum throughput. 

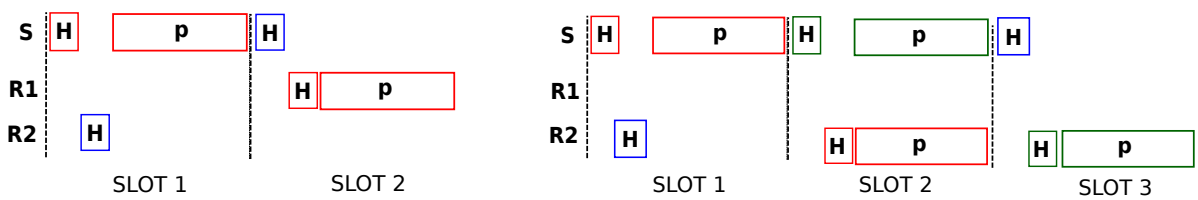

(a) State 0 (half-capacity relaying)

(b) State 1 (2/3-capacity relaying)

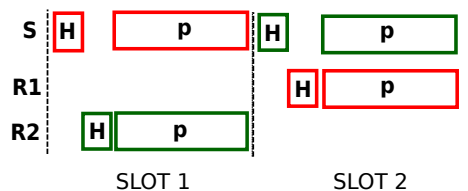

(c) State 2 (full-capacity relaying)

Figure 5: COLiDeR introduces 3 scheduling policies: the traditional half-capacity one-path relaying (5(a)); 2/3capacity two-path relaying (5(b)); and full-capacity two-path relaying (5(c)).

\subsubsection{States 1 and 1'}

$R_{1}$ can handle the reception of two signals while $R_{2}$ cannot (state 1 ) or $R_{2}$ can handle the reception of two signals while $R_{1}$ cannot (state 1') - areas A or B in Fig. 4 for one of the relays and area $\mathrm{C}$ for the other.

In this case, while full-capacity two-path relaying is not possible, there is a possibility for more simultaneous transmissions when compared to one-path relaying. Thus, COLiDeR introduces the concept of 2/3-capacity two-path relaying. To illustrate it, let us consider the case in which $R_{1}$ can handle the reception of two signals while $R_{2}$ cannot (State 1), as depicted in Fig. 5(b). In slot 1 , the source, $S$, transmits an interference-free packet to $R_{2}$. In slot $2, R_{2}$ relays the packet to the destination while at the same time $S$ transmits a second packet to $R_{1}$. Finally, in slot 3 , $R_{1}$ transmits the second packet, interference-free, to the destination, which ends up receiving two packets over 3 time slots (0.66 packets/slot) - a 2/3-capacity relaying.

\subsubsection{State 2}

Both relays can decode packets transmitted by the source while the other is transmitting areas A or B in Fig. 4 for the two relays. In this case, COLiDeR switches to full-capacity two-path relaying, as depicted in Fig. 5(c). The source transmits 1 packet per slot while either $R_{1}$ or $R_{2}$ relays a packet to the destination, which receives 1 packet/slot. 


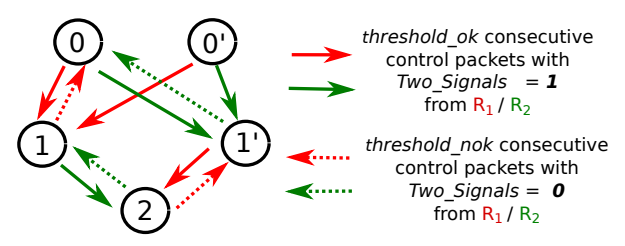

Figure 6: Source rate adaptive protocol state machine

\subsection{Distributed state transition}

In this section, we describe how COLiDeR addresses the challenge of identifying the proper two-path relaying state out of the 5 possible introduced in $\S 3.1$. What makes this task challenging is that the state depends on the intra-diamond state. Not only this information is not known in advance by any of the stations, but it can be subject to change due to the dynamic nature of the wireless channel.

At the high level, COLiDeR's approach is to have the source drive the transitions, with input from relays which are in the best position to estimate the best decoding strategy. The information necessary for the coordination is shared via the transmission of orthogonal control packets $(\mathrm{H}$ packets in Fig. 5), whose detailed format is presented in $\S 3.3$ (Fig. 7). We use the finite-state machine, depicted in Fig. 6, to model the selection of the two-path relaying state. The initial state, as well as the conditions for each transition, are as follows:

Initial state: To bootstrap, COLiDeR uses the traditional single-relay, half-capacity forwarding of packets. Either $R_{1}$ (state 0 ) or $R_{2}$ (state 0 ') exclusively forwards the packets from the source to the destination.

Transitioning to a higher state: The source and two relays periodically transmit control packets, as depicted in Fig. 5 . Leveraging the reception of these packets, $R_{1}$ (resp. $R_{2}$ ), can estimate $S N R_{S, R_{1}}$ and $S N R_{R_{2}, R_{1}}$ (resp. $S N R_{S, R_{2}}$ and $S N R_{R_{1}, R_{2}}$ ), necessary for deciding in which decoding area of Fig. 4 it most likely is. If $R_{1}$ estimates that decoding two signals is possible, i.e. area $\mathrm{A}$ or $\mathrm{B}$, it notifies the source by setting Two_Signals $=1$ in the next control packet it transmits. The source, based on the Two_Signals field of the control packets it receives, decides whether to transition to a higher state. To avoid overreacting to what could be a momentary change, the source only moves to a higher state if it receives a given number, threshold_ok, of consecutive packets with Two_Signals $=1$.

To elucidate, let us consider that COLiDeR is in state 0 and $R_{2}$, based on its analysis of the 


\begin{tabular}{|c|c|c|c|c|c|c|c|c|}
\multicolumn{1}{c}{ Length } & \multicolumn{1}{c}{$\begin{array}{c}\text { Next } \\
\text { State }\end{array}$} & \multicolumn{3}{c|}{$\begin{array}{c}\text { Power } \\
\text { Reduction }\end{array}$} \\
\hline $\begin{array}{c}2 \text { OFDM } \\
\text { symbols }\end{array}$ & $16 \mathrm{~b}$ & $2 \mathrm{~b}$ & $27 \mathrm{~b}$ & $2 \mathrm{~b}$ & $2 \mathrm{~b}$ & $1 \mathrm{~b}$ & $8 \mathrm{~b}$ & $8 \mathrm{~b}$ \\
\hline $\begin{array}{c}\text { Pilot } \\
\text { Symbols }\end{array}$ & \multicolumn{1}{c}{$\begin{array}{c}\text { Next } \\
\text { Hop }\end{array}$} & \multicolumn{3}{c}{$\begin{array}{c}\text { Current } \\
\text { State }\end{array}$} & $\begin{array}{c}\text { Two } \\
\text { Signals }\end{array}$ & CRC \\
\hline
\end{tabular}

Figure 7: Control Packet Format

recently received control packets, considers that it can decode packets sent by the source while $R_{1}$ is transmitting. To notify the source, $R_{2}$ sets the Two_Signals value as 1 in the next control packets it sends out. Upon receiving a given number, threshold_ok, of consecutive control packets with from $R_{2}$ with Two_Signals $=1$, the source decides to transition to state 1'. While still remaining in state 0 , it modifies the Next_State value in the following control packet it sends out, notifying the relays to immediately transition to state $1^{\prime}$. In the following slot the source transitions to state $1^{\prime}$ as well, completing the state transition, and modifies the Current_State field of the control packets it sends out accordingly. The transitions to states 1 and 2 follow a similar approach, with a detailed explanation omitted in the interest of brevity.

Transitioning to a lower state: The source will decide to shift to a lower state if one of the two events occur: a) it receives a given number, threshold_nok, of control packets with Two_Signals $=0$, or b) it receives no control packets that pass the CRC check for a given number, crc_threshold, of cycles. Should that happen, the source executes a state change by modifying the Next_State and Current_State fields in the control packets it sends out, similarly to the transition to higher state.

To account for the fact that packet losses are inevitable, the relays will transition by themselves to the initial state and send out control packets with Two_Signals $=0$ if they receive no control packets from the source passing the CRC check for a given number, crc_threshold, of cycles.

\subsection{Control packet format}

Figure 7 outlines COLiDeR's control packet format, which contains the following fields (sizes in bits):

1. Pilot_Symbols: Used for channel estimation in order to decode the rest of the control packet and, potentially, the following payload.

2. Length: Length of the following payload.

3. Next_hop: Next hop of the following payload. 
4. ID: 24 bits for the 3 packet IDs (with PLNC and partial linear combination reduction at relays, each payload may be a combination of at most 3 native packets) and 1 bit each to indicate whether of the native packet or the rotated packet is in the linear combination.

5. Current_State: State of the diamond.

6. Next_State: State to be adopted in the subsequent slot

7. Two_Signals: It is set to 0 or 1 , depending on the ability of the relays to handle the simultaneous reception of the packet sent by the source and the packet from the other relay.

8. Power_Reduction: Value in $\mathrm{dB}$ of the optional power reduction (see $\S 3.5$ ).

9. $C R C$ : Cyclic Redundancy Check in order to control the integrity of the received control packet.

\subsection{Knowing the $A B C-A$ measurement-driven approach}

In this section, we quantify the boundaries between decoding areas A, B, C using a measurementdriven approach. First, we start with the observation that the A-B and B-C boundaries are distinct in nature. Crossing from A to B, and vice versa, does not change a relay's capability to handle two overlapping signals, meaning this boundary is irrelevant to the source node (in both cases the relay sets Two_Signals $=1$ ). The relay, however, needs to know the boundary as it uses different decoding strategies depending on whether it is in A or B. Crossing from B to C, on the other hand, causes a relay to lose its ability to handle two overlapping signals, which it will report by changing the value of Two_Signals from 1 to 0 . What is more, such boundaries cannot obviously be clear-cut: one can be very conservative and make area $\mathrm{C}$ very large, at the cost of often being in states $0 / 0^{\prime}$, or pursue the full-capacity performance of state 2 by making $\mathrm{C}$ very small and $\mathrm{B}$ very large, at the cost of higher packet losses. COLiDeR captures this inherent trade-off by introducing two parameters $-\alpha$ and $\beta$ - as shown in Fig. 4. Formally, $\alpha$ and $\beta$ represent the values (in $\mathrm{dB}$ ) of interfering signals power difference, defining the boundaries of $\mathrm{A}, \mathrm{B}$ and $\mathrm{C}$ areas. In the following, we rely on measurements on hardware to identify the values of these two parameters that strike the best balance between throughput and packet losses.

\subsection{1. $\alpha$ selection}

To determine the best value for $\alpha$, we simply refer to the performance comparison conducted in $\S 2.3$. Figure 3 shows that when $S N R_{S, R_{1}}$ is $4 \mathrm{~dB}$ higher or more than $S N R_{R_{2}, R_{1}}$ IF decoding leads to a better Bit Error Rate than PLNC decoding. As a result, we adopt $\alpha=4 d B$. 
Table 1: Links state in the $\beta$ evaluation

\begin{tabular}{|c|c|}
\hline$S N R_{S, R_{1}}$ & $28+G d B$ \\
\hline$S N R_{S, R_{2}}$ & $31+G d B$ \\
\hline$S N R_{R_{1}, R_{2}}$ & $40 d B$ \\
\hline$S N R_{R_{2}, R_{1}}$ & $43 d B$ \\
\hline$S N R_{R_{1}, D} S N R_{R_{2}, D}$ & $>40 d B$ \\
\hline
\end{tabular}

\subsection{2. $\beta$ parameter selection}

As mentioned at the beginning of this section, when a relay crosses from area $\mathrm{B}$ to $\mathrm{C}$, it estimates that it cannot handle two signals anymore and changes Two_Signals from 1 to 0 . It leads the source station to transition to a lower state (Cf. § 3.2$)$ and the relay itself to switch to IF decoding. As a result, a low $\beta$ value will cause the system to be in the low two-path relaying states, reducing the maximum throughput, while a large $\beta$ value will lead to an aggressive pursuit of the higher states, with the higher maximum throughput, but at the cost of higher packet losses.

With the $\beta$ parameter impacting the throughput and packet delivery rate, in the following we evaluate its impact on both these metrics. We place the 4 USRPs of our testbed so as to obtain the SNRs values indicated in Table 1 . For three different values of $\beta$ we carry a series of experiments in which we change the source transmission gain $G$, which directly affects $S N R_{S, R_{1}}$ and $S N R_{S, R_{2}}$. We assume the source always has packets to transmit.

Figure 8 reveals an interesting trade-off between throughput and packet delivery rate induced by the choice of $\beta$. When its value is highest, $15 d B$, relays are always in area $\mathrm{B}$, leading the source to always maintain state 2 , resulting in the highest performance in terms of throughput, as illustrated in Fig.8(a). However, because the relays overestimate their real ability to decode two interfering packets, this comes at a high cost in terms of packet losses, as Fig. 8(b) shows. On the other side of the spectrum, with $\beta=3 d B$, the source remains in state 0 . Even if very few packets are lost, the achieved throughput in this case does not exceed 0.5 frames per slot. Setting $\beta=9 d B$ strikes the best trade-off: the throughput is never more than $15 \%$ lower than with $\beta=15 d B$ but the number of lost packets is considerably reduced. Thus, it is the value adopted by COLiDeR. 


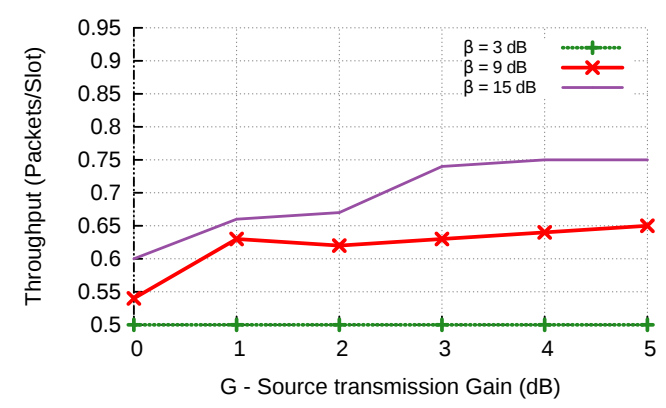

(a) Received Throughput

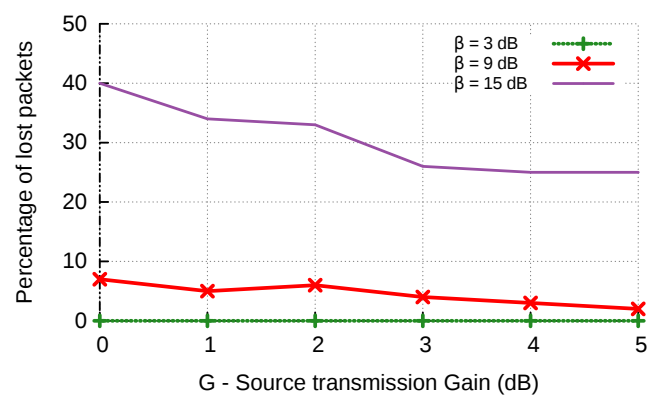

(b) Lost packets

Figure 8: Trade-off between the received throughput and the percentage of lost packets

\subsection{Optional source power control}

Our study for identifying the best values for $\beta$ revealed that the system performance is still impacted by the intra-diamond channel. For example, Fig. 8(a) shows that the throughput never reaches the expected maximum throughput of 1 packet/slot. This is due to the fact that the performance of PLNC decoding can suffer under particular channel conditions. As Fig. 3 shows, the BER obtained with PLNC decoding presents two local peaks when $\left|S N R_{S, R_{1}}-S N R_{R_{2}, R_{1}}\right|=3 d B$. These particular BER variations values are not coincidental or result of particular hardware but rather represent singularities inherent to how PLNC decoding works. As detailed in [11], they are due to a reduction in the distance between the received constellation points occurring when $\theta$ approaches $\frac{\Pi}{2}$.

Therefore, a clear path to improving performance is adjusting the transmit power levels so as to steer COLiDeR towards the BER local minima observed when $S N R_{S, R_{1}}-S N R_{R_{2}, R_{1}}$ is $0 d B$ or $-7 d B$ (Fig. 3). The simplest option would be to adjust the transmit power of all three nodes, however, this could have unintended consequences on the relay-destination links that we consider strong in this work. Thus, we adopt an approach in which only the source transmission power is adjusted. The basic idea is for the relays to report to the source the SNR difference between the signal from the source and the signal from the other relay and for the source to adjust its transmit power as necessary. When a relay, say $R_{1}$, estimates that the power difference between the two signals is close to $3 d B$ (resp. $-3 d B$ ), it asks to the source to reduce its transmission power by a value indicated in the dedicated field of each control packet such that $S N R_{S, R_{1}}-S N R_{R_{2}, R_{1}}=0 d B$ (resp. $S N R_{S, R_{1}}-S N R_{R_{2}, R_{1}}=-7 d B$ ). 


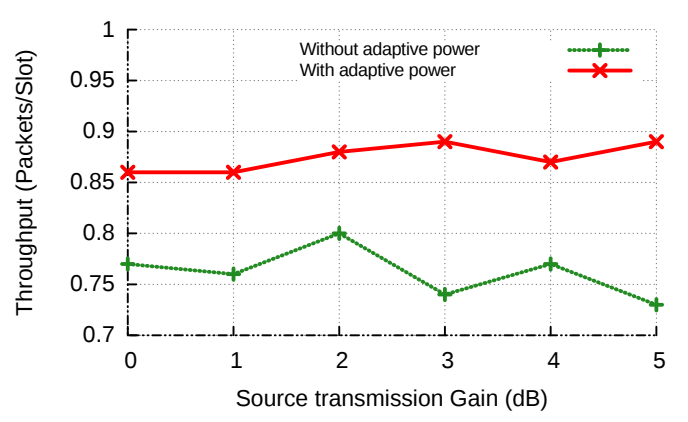

Figure 9: Power adaptation mechanism evaluation

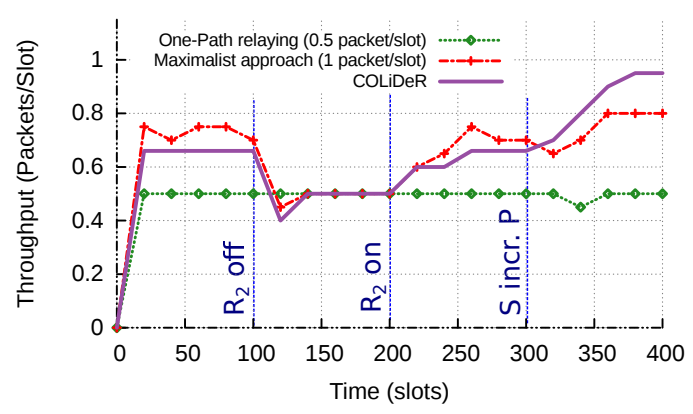

(a) Received Throughput

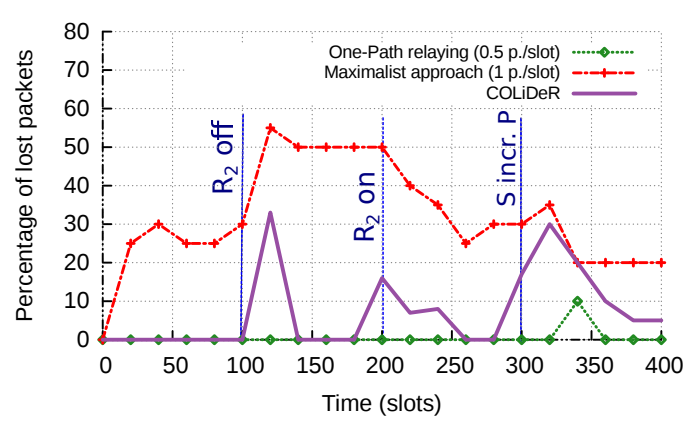

(b) Lost packets

Figure 10: COLiDeR performance in a general evaluation scenario

In order to evaluate the gains of the proposed power adaptation mechanism, we place our USRP nodes with the aim of having COLiDeR transition to state 2 and both relays using PLNC decoding (area B). We then vary the maximum transmission power of the source node by varying the transmission gain $G$ and evaluate the number of packets received at the destination with and without the power adaptation mechanism. As shown in Fig. 9, when using power control, COLiDeR's performance never drops below 0.85 frames/slot, a $15 \%$ improvement, on average and with no impact on packet loss.

\subsection{General evaluation}

Method: To evaluate the overall performance of COLiDeR in different channel conditions, we implement a scenario incorporating four representative configurations. Initially, configuration $I$, the 4 USRP radios are placed in the classic diamond topology and the transmission power levels are selected such that only one relay can handle two simultaneous transmissions with a reasonable bit error rate. At time $t_{0}=100$ slots, relay $R_{2}$ is switched off, creating configuration $t_{0}$ aimed at 
emulating a relay loss due to mobility. At $t_{1}=200$ slots, relay $R_{2}$ is switched back on, emulating the selection of a new relay. In the final configuration, at $t_{2}=300$ slots, the source transmission power is increased, emulating a scenario in which the source moves closer to the relays.

Comparison: Our goal is to evaluate how COLiDeR navigates the trade-off between reliability and throughput, its principle design consideration. Note that COLiDeR could optimize reliability by permanently staying in state 0 , the equivalent of traditional one-path relaying. Thus, this approach is included in the study. On the opposite side, having COLiDeR permanently in state 2 maximizes throughput even though it may cause severe packet losses. To see why this is true, consider the case in which $R_{1}$ and $R_{2}$ can not handle the reception of two interfering signals. While $R_{1}$ is transmitting a packet to the destination, the source has two choices. It can transmit to $R_{2}$, the maximalist approach, leading to a packet loss. Or it does not transmit, which is what COLiDeR would have done since it would have transitioned to state 0 . In terms of throughput, assuming layer two will retransmit the same packet in the following slot, the maximalist approach did no worse than COLiDeR even though it transmitted unnecessarily, wasting energy and augmenting interference. COLiDeR, however, may sometimes do worse than the maximalist approach in terms of throughput, either because it is designed to wait for a few rounds before it transitions to the next best state, or because it chooses to be in a lower state to avoid packet losses. Thus, the maximalist approach provides a good reference to compare against.

Results: Figure 10 plots the end-to-end throughput (Fig. 10(a)) and percentage of lost packets (Fig. 10(b)) against time. Each value is obtained by computing the throughput and loss ratio over the 30 previous slots.

In the initial configuration, $I, R_{1}$ can handle the reception of two signals with a reasonable bit error rate whereas $R_{2}$ cannot. COLiDeR adopts scheduling state 1 ( $\S 3.1$ ), resulting in 0.66 packets/slot throughput and no packet losses - the best joint throughput-reliability performance. When the source adopts the maximalist approach and transmits a packet every slot, a slightly higher (around $8 \%$ ) throughput is achieved, because occasionally, $R_{2}$ successfully decodes the interfering packets. It comes, however, with a high penalty in terms of packet losses, around $25 \%$. Furthermore, COLiDeR performs just as well as one-path relaying in terms of packet losses while outperforming it by $25 \%$ in terms of throughput.

At $t_{0}=100$ slots $R_{2}$ is switched off, leaving a single path to the destination. Obviously, as 
Fig. 10(a) shows, the maximum throughput in this case, realized by all schemes, is 0.5 packet/slot. Having the source sending 1 packet/slot when only $R_{1}$ is active, however, leads to an inevitable $50 \%$ rate loss. As described in $\S 3.2, \mathrm{COLiDeR}$, after receiving no control packets from $R_{2}$, transitions from state 1 to state 0 , limiting the packet losses.

At $t_{1}=200$ slots, relay $R_{2}$ is switched back on and is eventually integrated back to two-path relaying, enabling the two-path relaying schemes to realize the performance observed in the period 0-100 slots. Finally, at $t_{2}=300$ slots the source transmission power is increased, enabling $R_{2}$ to handle the reception of two simultaneous signals with a reasonable bit error rate. COLiDeR takes advantage of the newly created opportunity and transitions to state 2 , achieving close to channelcapacity performance in terms of throughput with almost zero packet losses. The maximalist approach, while also transmitting 1 packet/slot, does not include power control, explaining its inferior performance when compared to COLiDeR.

\section{COLiDeR in multi-hop wireless networks}

In the previous sections, in order to design COLiDeR, we considered the 4 nodes involved in the relaying process in isolation. The question we consider in this section is how to integrate our scheme in larger networks, in particular in multihop wireless networks where tens of flows may be established simultaneously. In these networks, many nodes may be competing with each other for channel access. As a result, integrating COLiDeR is not straightforward: it requires a specific channel access mechanism for the 4 nodes to reserve the channel while causing all the potential interfering neighbors to defer. In this section, we address this challenge by introducing and evaluating an 802.11-inspired channel access method.

\subsection{COLiDeR-adapted access method}

While a large panel of channel reservation mechanisms for multihop wireless networks has been proposed in the literature, we choose a TDMA-based MAC layer as reference since COLiDeR is especially designed for time-synchronized devices. In particular, we focus on a slotted version of the 802.11 DCF protocol that has drawn the attention of the research community in the last decade [15] [16] [17]. In this channel reservation method, each frame is composed of (i) a data slot dedicated to data transmissions and (ii) a contention slot for allocating the channel in a distributed manner. As illustrated in Fig 11, the contention part is divided into $M$ mini-slots, in which nodes 


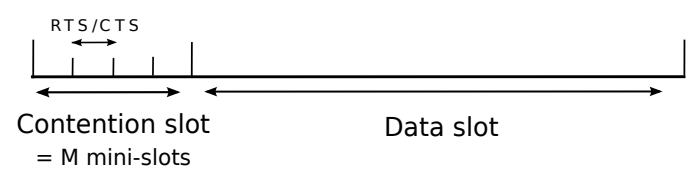

Figure 11: Frame: contention slot + data slot

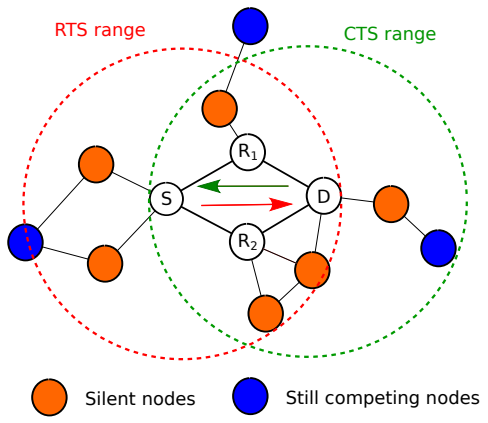

Figure 12: Two-path relaying channel reservation

contend to gain access to the immediately following data transmission part. In each mini-slot, a node wanting to transmit a packet to one of its neighbors (it may be the final destination or only the next hop of a particular flow) and which has not overheard any signal in the previous mini-slots of the same frame, sends an RTS packet with a probability $\alpha$. In the second part of the same mini-slot, the intended receiver sends back a CTS packet, if it has not overheard any signal in the previous mini-slots either. If the RTS/CTS exchange is successful, the source node is allowed to transmit its data packet in the next data slot. Otherwise, it remains silent and waits for the next contention slot to attempt again. With such a mechanism, the resulting schedule of the data slot is interference-free and the transmitted packets are correctly received by their receivers (we do not consider in this work the errors inherent to wireless transmissions).

However, this scheduling mechanism is designed for 1-hop interference-free transmissions. As illustrated in Fig. 12, we propose some modifications to integrate two-path relaying while keeping the same main design principles.

1. RTS/CTS transmission range: Whereas the original version leverages the 1-hop transmission range for both control and data packets, we increase the transmission range of RTS/CTS packets in order for the senders to reach their 2-hop neighbors. In practice, this can be done by increasing power transmission or by adopting a more robust Modulation-Coding Scheme (MCS). With this, each source of a two-path relaying process can send directly its RTS packet to the 


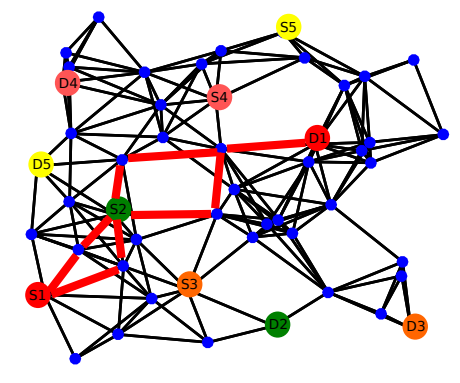

(a) Scenario 1

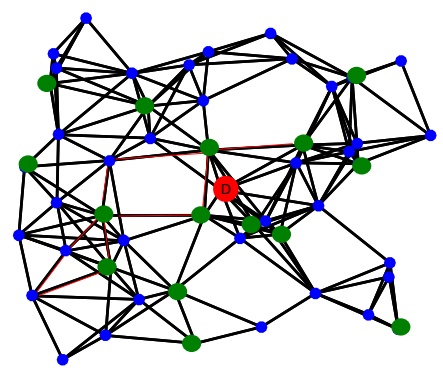

(b) Scenario 2

Figure 13: COLiDeR performance in a 50-node scenario

destination of the same diamond (and the destination can reply with a direct CTS).

2. RTS/CTS content: In the original version, RTS/CTS packets contain only the ID of the source and destination nodes. We modify the packets to also include the ID of the two involved relays. As a result, all the neighbors receiving RTS/CTS packets know exactly which nodes aim to access the channel in the next data slot. Note that, for traditional 1-hop reservation (when no two-path relaying opportunity exists), the RTS/CTS packet remains the same.

3. Channel access contention: Whereas in the original protocol, a node $n$ stops participating in the access race as soon as it overhears a signal from one of its neighbors, in our COLiDeRadapted mechanism it stops only if one the ID indicated in the control packets identifies a 1-hop neighbor of $n$. For instance, in Fig. 12, the blue nodes stay in the competition for access after receiving the RTS/CTS packets sent by the source and the destination of the established diamond. Such an adaptation aims to reduce the interference footprint of the 2-hop RTS/CTS transmissions.

\subsection{Performance in multi-hop wireless networks}

To evaluate the performance of the proposed COLiDeR-adapted scheduling mechanism, we have developed our own discrete-event simulator in MATLAB that runs both the original interferencefree and COLiDeR-adapted mechanisms. We carried experiments on the 50-node topology shown in Fig. 13 and present here the results for two particular scenarios, relying on two different communication patterns. 


\subsubsection{Scenario 1: decentralized communications}

Simulation description: In the first scenario, we instantiate 5 flows by selecting 5 different source-destination pairs, as illustrated in Fig. 13(a). Each path is randomly selected among the available shortest paths (in terms of hops) between the source and the destination. In the COLiDeR-adapted scheduling case, the nodes (sources and relays) always leverage two-path relaying opportunities when they occur (nodes know their 2-hop neighborhood and the two-path opportunities for each flow). As a consequence, as illustrated for the flow 1 in Fig. 13(a), paths may be composed of both traditional 1-hop link and COLiDeR's diamonds.

To ensure fairness in the evaluation, the scheduling parameters are the same for the interferencefree and the COLiDeR-adapted access methods: the contention slots are composed of 2 mini-slots $(M=2)$ in order to limit the overhead and the probability of transmission alpha is set to 0.02 in order to reduce collisions during the access race. A lot of algorithms have been developed in the last years in order to optimize these parameters [16] [17]. However, this is beyond the scope of this paper and we will use the same constant value for all the nodes of the network. Last but not least, we assume that the selected diamonds are in state 2 of COLiDeR, meaning that the relays can receive a packet from the diamond source when the other relay is transmitting. In other words, the diamonds have the same capacity as the traditional 1-hop links.

Results: As performance metric, we evaluate the average number of packets received by the 5 final destinations during 4000 slots over 500 simulation runs. We work at saturation: the 5 sources always have a packet to transmit in their queue. Fig. 14 shows that the integration of COLiDeR in this multi-hop wireless network increases the overall throughput, since the number of received packets is higher when two-path relaying is activated. Indeed, since COLiDeR triggers two transmissions instead of one, as is the case with the original interference-free reservation mode, more transmissions are established in a single frame and packets reach faster their destination.

\subsubsection{Scenario 2: centralized communications}

Simulation description: In the second scenario, we keep the same network topology but we change the communication pattern. Now, there is only one destination (red node in Fig. 13(b)) and 15 nodes (green nodes in Fig. 13(b)) want to send data to this node. This is representative of an IoT network in which multiple sensors transmit their packets to a central 


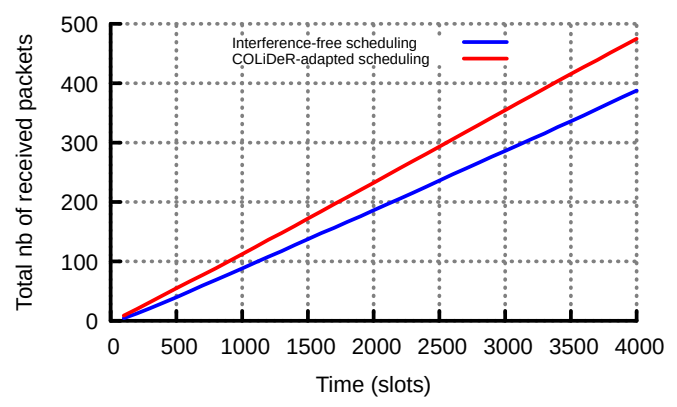

Figure 14: Scenario 1: Number of received packets during 4000 slots of simulation

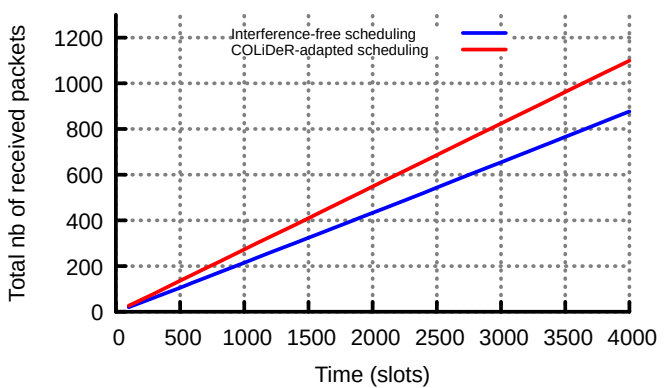

(a) Total number of received packets

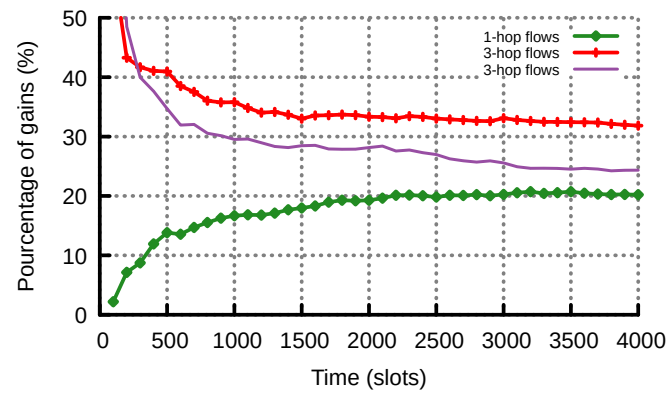

(b) Throughput gains depending on the number of hops

Figure 15: Scenario 2: Performance of COLiDeR

gateway. Otherwise, the scheduling settings are the same than in previous simulations $(M=2$ and $\alpha=0.02)$.

Results: As for decentralized communications, the total number of packets received by the central destination increases when two-path relaying is allowed (Fig. 15(a)). To refine and complement this result, we also evaluate in Fig. 15(b) the average throughput gain of two-path relaying compared to traditional interference-free transmissions as function of the number of hops between the source and the destination. As expected, the data shows that 2-hop flows achieved the highest gains (more than 30\% after 4000 slots of simulation) since each path involved one diamond. Surprisingly, however, the data shows a throughput gain of $20 \%$ by the end of the simulation for one-hop flows. This can be explained by the fact that two and 3-hop flows are transmitted faster to the destination, leaving a larger share of the channel capacity to the one-hop flows. 


\section{Related Work}

Due to its potential for minimizing the multiplexing loss using half-duplex radios, the twopath relaying has attracted a lot of attention in recent years. First, Fan et al. introduced a scheduling policy capable of eliminating the multiplexing loss, enabling the destination to receive 1 packet/slot [4]. However, this solution assumes optimal decoding of the two overlapping signals at the relays. Its performance drops significantly when realistic decoding errors are introduced [18]. As our work has shown, two-path relying is founded on the capability of modern radios to handle two overlapping transmissions, making the choice of the decoding strategy of paramount importance. In [5] and [6], Successive Interference Cancellation (SIC) [10] was advanced as the best potential candidate. Nevertheless, it is noted that depending on the interfering signals power levels, SIC can be highly inefficient. Our work is the first to address this oversight and present a complete solution combining Physical-Layer Network Coding and interference-free equalization. A two-path relaying approach that relaxes the assumption of optimal decoding is proposed in [7]. The main idea is to fall back to one-path relaying when two-path relaying suffers from too many decoding errors. However, this work is mostly theoretical and leaves unaddressed many crucial practical issues such as how does the source know of the decoding errors in the first place. COLiDeR is the first protocol that addresses the challenges arising from realizing two-path relaying in practice.

\section{Conclusion and Future Work}

We have presented COLiDeR a PHY/MAC cross-layer solution for adaptive two-path relaying. Our solution addresses the 3 main challenges for the integration of two-path relaying in multihop wireless networks. First, our experimentally driven approach leverages real testbed evaluations for identifying the best interference management technique to handle colliding packets (PHY-challenge). Our experiments highlight the inefficiency of Self Interference-free Cancellation, leveled in its recommended area of operation by the Physical-Layer Network Coding and outperformed elsewhere by either PLNC or basic equalization. Then, COLiDeR includes a relaying protocol that selects dynamically the optimal scheduling scheme depending on channel state and topology evolution (REL-challenge). Optionally, our protocol integrates power control that leverages singularities in how PLNC works for increased efficiency. Based on a well defined control plane messages and an efficient setting of the thresholds that our solution relies on, COLiDeR 
ensures smooth and efficient sailing between the defined protocol states. Experimental results have shown that COLiDeR achieves between $80-95 \%$ of the relaying performance of the ideal fullduplex radio while incurring negligible decoding failures. Finally, on the top of COLiDeR, we also propose a fully-distributed access method in order to integrate it in multihop wireless networks (MAC-challenge). We have integrated this mechanism in an discrete-event simulator and the obtained results have shown that COLiDeR may increase the overall throughput by $20 \%$ in a 50 -node networks, with multiple flows and different traffic patterns.

In the future, we intend to work on two different problems in order to extend the sphere of operation of COLiDeR. First, in this paper we leverage on measurement-driven approaches to select the optimal values of the defined parameters. We believe that these values could be adapted dynamically depending on the observed channel conditions, thanks to Deep or Reinforcement Learning techniques. Then, while this work paves the way to the integration of COLiDeR in multihop wireless networks, two-path relaying needs other higher layer protocols to fully efficient in these networks. Many questions could be of interest: How to identify diamonds with a limited 1hop topology knowledge? Can we override the next hop decisions imposed by the routing protocol in order to have the required flexibility in the relay selection?

\section{References}

[1] R. Naves, G. Jakllari, H. Khalifé, V. Conan, A.-L. Beylot, Colider : A cross-layer protocol for two-path relaying, in: ACM MSWiM 2019, p. 247-251.

[2] L. Chen, F. Wu, J. Xu, K. Srinivasan, N. Shroff, Bipass: Enabling end-to-end full duplex, in: ACM Mobicom 2017, Snowbird, Utah, USA, pp. 114-126.

[3] D. Bharadia, S. Katti, Fastforward: Fast and constructive full duplex relays, in: ACM SIGCOMM 2014, Chicago, Illinois, USA, pp. 199-210.

[4] Y. Fan, C. Wang, J. Thompson, H. V. Poor, Recovering multiplexing loss through successive relaying using repetition coding, IEEE Transactions on Wireless Communications, Vol. 6, No. 12 (2007).

[5] C. Zhai, W. Zhang, P. C. Ching, Cooperative spectrum sharing based on two-path successive relaying, IEEE Transactions on Communications, Vol. 61, No. 6 (2013).

[6] R. Simoni, V. Jamali, N. Zlatanov, R. Schober, L. Pierucci, R. Fantacci, Buffer-aided diamond relay network with block fading and inter-relay interference, IEEE Transactions on Wireless Communications, Vol. 15, No. 11 (2016).

[7] F. Tian, W. Zhang, W.-K. Ma, P. Ching, H. V. Poor, An effective distributed space-time code for two-path successive relay network, IEEE Transactions on Communications, Vol. 59, No. 8 (2016). 
[8] S. Zhang, S. Liew, P. Lam, Hot topic: physical-layer network coding, in: Proc. ACM MobiCom Conference, 2006.

[9] Gnuradio overview available at http://www.gnuradio.org/.

[10] D. Halperin, T. Anderson, D. Wetherall, Taking the sting out of carrier sense: Interference cancellation for wireless lans, in: Proc. IEEE MOBICOM Conference, 2008.

[11] T. Koike-Akino, P. Popovski, V. Tarokh, Optimzed constellations for two-way wireless relaying with physical network coding, IEEE Journal On Selected Areas in Communications, Vol. 27, No. 5 (2009).

[12] V. N. V. T. Muralidharan, B. Rajan, Wireless network-coded bidirectional relaying using latin squares for m-psk modulation, IEEE Transactions On Information Theory, Vol. 59, No. 10 (2013).

[13] L. You, S. Liew, L. Lu, Network-coded multiple access ii: Toward real-time operation with improved performance, IEEE Journal On Selected Areas in Communications, Vol. 33, No. 2 (2015).

[14] T. Hynek, D. Halls, J. Sykora, Hardware implementation of distributed learning algorithm for mapping selection for wireless physical layer network coding, in: Proc. IEEE ICC Workshop (ICCW) 2015, 2015.

[15] A. Gupta, X. Lin, R. Srikant, Low-complexity distributed scheduling algorithms for wireless networks, IEEE/ACM Transactions on Networking 17 (6) (2009) 1846 - 1859

[16] J. Ni, B. Tan, R. Srikant, Q-csma: Queue-length-based csma/ca algorithms for achieving maximum throughput and low delay in wireless networks, IEEE Transactions on Networking, Vol. 20, No. 3 (2012).

[17] C. Joo, M. Shin, Queue-affectance-based scheduling in multi-hop wireless networks under sinr interference constraints, in: IEEE INFOCOM Conference 2016, 2016.

[18] Q. Li, M. Yu, A. Pandharipande, X. Ge, Outage analysis of co-operative two-path relay channel, IEEE Transactions On Wireless Communications, Vol. 15, No. 5 (2016). 